\title{
Gender Difference in Associations Between Telomere Length and Risk Factors in Patients With Stroke
}

\begin{abstract}
Yuqing Wang ${ }^{1,2+}$, Fengjuan Jiao ${ }^{2 \dagger}$, Huancheng Zheng ${ }^{1,2}$, Qingsheng Kong ${ }^{3}$, Ran $\mathrm{Li}^{1}$, Xiaojie Zhang ${ }^{4,5}$, Li Yan ${ }^{6}$, Yanlei Hao ${ }^{7 *}$ and Yili Wu ${ }^{8,9,10,11 *}$

${ }^{1}$ Cheeloo College of Medicine, Shandong University, Jinan, China, ${ }^{2}$ Shandong Collaborative Innovation Center for Diagnosis, Treatment and Behavioral Interventions of Mental Disorders, Institute of Mental Health, Jining Medical University, Jining, China, ${ }^{3}$ Collaborative Innovation Center for Birth Defect Research and Transformation of Shandong Province, Jining Medical University, Jining, China, ${ }^{4}$ Department of Psychiatry, The Second Xiangya Hospital, Central South University, Changsha, China, ${ }^{5}$ National Clinical Research Center for Mental Disorders, Changsha, China, ${ }^{6}$ Department of Neurology, China-Japan Friendship Hospital, Beijing, China, ${ }^{7}$ Department of Neurology, Affiliated Hospital of Jining Medical University, Jining, China, ${ }^{8}$ Key Laboratory of Alzheimer's Disease of Zhejiang Province, Institute of Aging, School of Mental Health, Wenzhou Medical University, Wenzhou, China, ${ }^{9}$ The Affiliated Kangning Hospital, Wenzhou Medical University, Wenzhou, China, ${ }^{10}$ Oujiang Laboratory, Wenzhou, China, ${ }^{11}$ Shandong Collaborative Innovation Center for Diagnosis, Treatment and Behavioral Interventions of Mental Disorders, Institute of Mental Health, Jining Medical University, Jining, China
\end{abstract}

Multiple risk factors of stroke are associated with telomere length shortening. Although leukocyte telomere length (LTL) is shorter in patients with stroke, the heterogeneity is high. Risk factors may be differentially associated with LTL in male and female patients contributing to the heterogeneity. However, the gender difference in associations between LTL and risk factors in stroke patients has not been investigated. In this study, we investigated the gender difference in associations between LTL and risk factors in 312 stroke patients. Real-time quantitative PCR was used to determine relative LTL, and multiple linear regression analysis was applied for association analyses. We found that LTL was negatively associated with triglyceride $(\mathrm{TG})$ in all patients $[\beta(95 \% \mathrm{Cl})=-0.69$ $(-1.26,-0.11), P<0.05]$ after adjusting confounders. Importantly, LTL was negatively associated with lack of exercise $[\beta(95 \% \mathrm{Cl})=-1.80(-3.12,-0.49), P<0.05]$ and LDL levels $[\beta(95 \% \mathrm{Cl})=-3.22(-6.05,-0.390), P<0.05]$ in male patients, while LTL was negatively associated with dyssomnia $[\beta(95 \% \mathrm{Cl})=-2.00(-3.96,-0.07), P<0.05]$ and diabetes $[\beta(95 \% \mathrm{Cl})=-2.13(-4.10,-0.27), P<0.01]$ in female patients. Our study showed that LTL is differently associated with risk factors in male and female patients with stroke, indicating that gender difference should be considered when LTL is potentially applied as an index of risk and prognosis for stroke. Our study also provides an insight into that gender differences should be considered when developing intervention strategies for stroke prevention and treatment.

Keywords: gender, risk factors, stroke, telomere length, gender difference

\section{INTRODUCTION}

Stroke, an age-related disease, is one of the leading causes of death and long-term disability worldwide, bringing a heavy burden to both family and society (Donnan et al., 2008). As an agerelated disease, the risk of stroke is increased with age (Calado and Young, 2009). In addition, risk factors including current smoking, alcohol intake, unhealthy diet, abdominal obesity, less physical 
activity, hypertension, and diabetes mellitus, etc., are associated with a $90 \%$ risk of stroke and prognosis of stroke (O'Donnell et al., 2010). Telomere length is declined with age in adults less than 75 years (Lapham et al., 2015). Overall telomere attrition predicts mortality and risk of aging-related diseases in general human cohorts (Blackburn et al., 2015). Consistently, leukocyte telomere length (LTL) shortening was associated with the risk of stroke (Kappei and Londoño-Vallejo, 2008; Luo et al., 2017; Gao et al., 2018; Jin et al., 2018; Tian et al., 2019). Moreover, stroke-related risk factors such as obesity, smoking, alcohol intake, psychological stress, and depression are linked to telomere length shortening (Tian et al., 2019). However, telomere length shortening does not have a causal effect on stroke based on Mendelian randomization studies (Cao et al., 2019, 2020). It suggests that LTL shortening might be the consequence of the pooled effect of risk factors. Thus, LTL shortening may serve as an index of stroke risk and potentially serve as an index of prognosis for stroke.

The heterogeneity of LTL is high in various studies, although LTL is short in patients with stroke in a meta-analysis (Jin et al., 2018; Tian et al., 2019). Gender difference may be a key factor for the heterogeneity as a gender-based difference of LTL is observed in the general population (Gardner et al., 2014; Needham et al., 2014; Lapham et al., 2015; Choi et al., 2018; Ghimire et al., 2019). Moreover, gender-specific associations between LTL and multiple factors including risk factors have been observed in the general population or in patients with coronary artery disease, an age-related disease (Cheng et al., 2017; Shadyab et al., 2017a,b; Ghimire et al., 2019; Sullivan et al., 2019). However, none of the aforementioned studies examined associations between LTL and risk factors in male and female stroke patients, respectively. In this study, we aimed to explore differential associations between risk factors and LTL in male and female stroke patients, respectively. It may provide an insight into that gender difference should be considered when LTL is potentially applied as an index of risk and prognosis for stroke and that gender difference should be considered when developing intervention strategies for stroke prevention and treatment.

\section{SUBJECTS AND METHODS}

\section{Participants}

Three hundred fifty-one patients were enrolled from the Affiliated Hospital of Jining Medical University between May 2016 and February 2017; 39 patients were excluded because of missing information or not meeting the inclusion criteria. Thus, 312 stroke patients were included in the final data analysis. Inclusion criteria: patients diagnosed with ischemic stroke (ICD10: 163.9) or diagnosed with temporary ischemic attacks (TIAs, ICD-10: G45.9) based on "Chinese guidelines for diagnosis and treatment of acute ischemic stroke (2010)"; the first-onset stroke or TIAs; and patients with consciousness. Exclusion criteria: patients with unconscious disorders, language disorders, dementia, neuropsychiatric, or severe complications. This is a cross-sectional study. Experiments were conducted according to the principles expressed in the Declaration of Helsinki.
This study was approved by the Research Ethics Committee of Jining Medical University. The written informed consents were obtained from patients or their guardians.

\section{Data Collection}

On the day of admission, the clinical data were collected by neurologists. The information of demographic characteristics, lifestyles, and disease history was collected and shown in Table 1. Subjects who smoked more than one cigarette/day for 1 year or above were defined as current smokers. Smokers who had quit smoking for more than half a year were defined as former smokers. Subjects who never smoke were defined as non-smokers. Subjects who drank more than one time/week for at least half a year were defined as current alcohol drinkers. Otherwise, subjects were defined as non-alcohol drinkers or occasional alcohol drinkers. Subjects who did physical exercise > $30 \mathrm{~min} /$ day were defined as exercise well. The quality of sleep was assessed according to "Guidelines for the diagnosis and treatment of adult insomnia in China (2017)." The patients with the following symptoms were diagnosed with dyssomnia: sleep latency of more than $30 \mathrm{~min}, \geq 2$ awakenings throughout the night, early-morning awakening, reduced sleep quantity, and/or less than $6.5 \mathrm{~h}$ sleep per night, as well as accompanied by excessive sleepiness during the daytime. The medication was collected and presented in Supplementary Table 1. Body mass index (BMI) was calculated as weight divided by squared height. The blood samples were collected within $72 \mathrm{~h}$ since the onset of stroke. Specimens were tested on a Roche cobas 8000 system (c701 and c702 modules). The following biochemical indexes, known as risk factors of stroke, were included for data analyses: fasting blood glucose (FBG; normal range value: $3.89 \sim 6.1 \mathrm{mmol} / \mathrm{L}$ ); triglyceride (TG; normal range value: $0.45 \sim 1.69 \mathrm{mmol} / \mathrm{L}$ ); total cholesterol (TC; normal range value: $3.00 \sim 5.69 \mathrm{mmol} / \mathrm{L}$ ); high-density lipoprotein: (HDL; normal range value: $0.70 \sim 2.00 \mathrm{mmol} / \mathrm{L}$ ); low-density lipoprotein (LDL; normal range value: $<3.12 \mathrm{mmol} / \mathrm{L}$ ); and very low-density lipoprotein (VLDL; normal range value: $0.21 \sim 0.78 \mathrm{mmol} / \mathrm{L})$. The data of biochemical indexes were presented in Table 1.

\section{The Relative Telomere Length Measurement}

Genomic DNA was extracted from whole blood samples using the RelaxGene Blood DNA System (Tiangen Biotech, Co., Ltd., Beijing, China) according to the instructions of the manufacturer and stored in a $-80^{\circ} \mathrm{C}$ freezer (Genetic Resource Center of Jining Medical University). Relative LTL was measured by real-time quantitative PCR (qPCR) (Cawthon, 2002). The gene 36B4 was used as a single copy reference gene to normalize the quantity of input DNA. The cycle threshold $(\mathrm{Ct})$ values were used to calculate the relative telomere length. The equation was $T / S=2^{-(\Delta \mathrm{Ct})}$, where $T=$ telomere repeat copy number, $S=36 \mathrm{~B} 4$ copy number, and $\Delta \mathrm{Ct}=\mathrm{Ct}$-telomere-Ct-36B4. All $T / S$ ratios of telomere DNA were normalized to the $T / S$ ratio of reference DNA. The primer sequences $\left(5^{\prime} \rightarrow 3^{\prime}\right)$ were tel1b: GGTTTTTGAGGGTGAGGGTGAGGGTGAGGGTGAGGGT, 
TABLE 1 | Characteristics of 312 stroke patients.

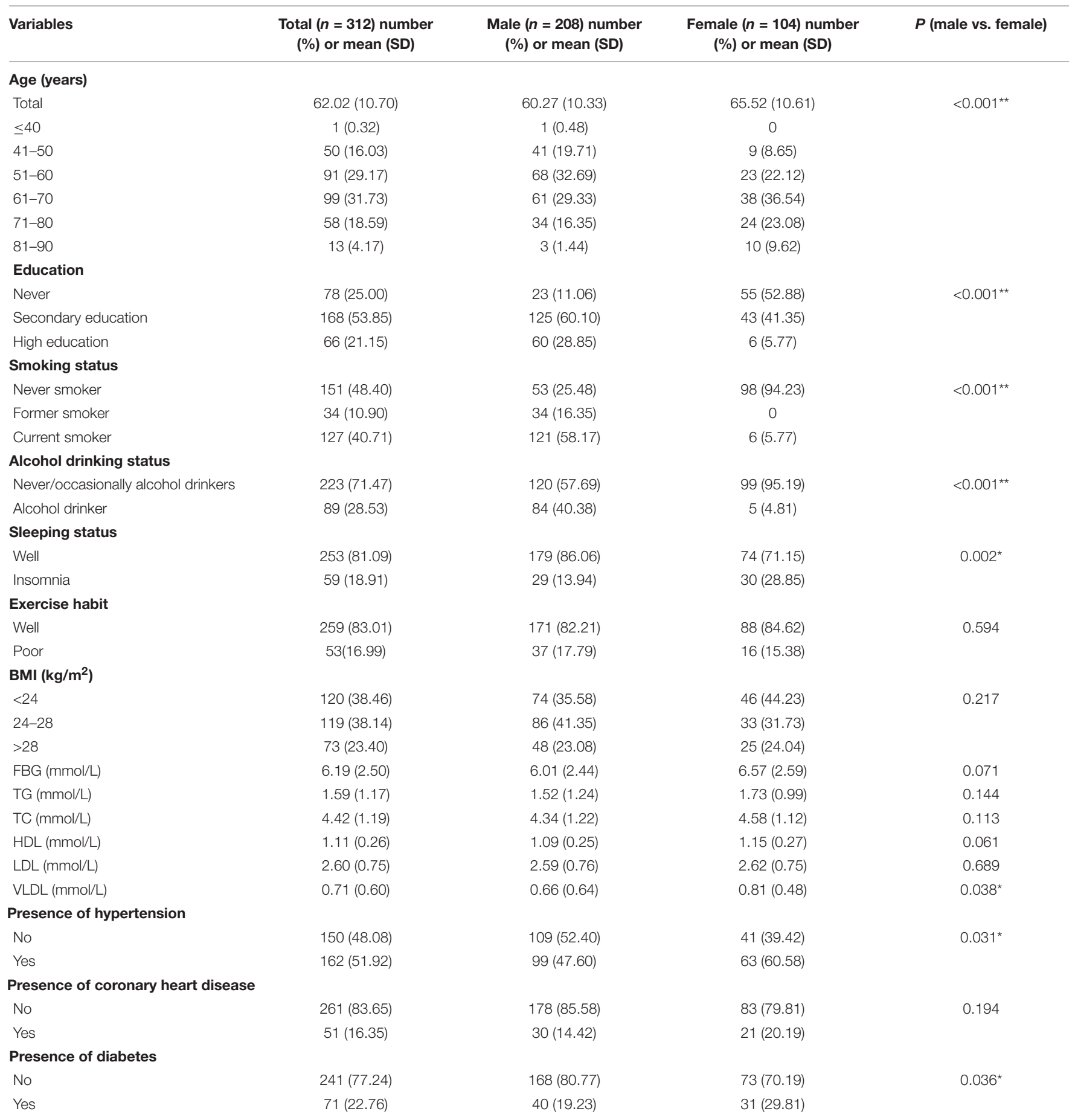

FBG, fasting blood glucose; TG, triglyceride; TC, total cholesterol; HDL, high-density lipoprotein; LDL, low-density lipoprotein; VLDL, very low-density lipoprotein. ${ }^{*} P<0.05 ;{ }^{* *} P<0.001$.

te12b: TCCCGACTATCCCTATCCCTATCCCTATCCCTATCCC TA, 36B4u: CAGCAAGTGGGAAGGTGTAATCC, and 36B4d: CCCATTCTATCATCAACGGGTACAA, respectively. The reaction system consisted of $10 \mu \mathrm{l}$ of MasterMix, $0.4 \mu \mathrm{l}$ of each primer, $2 \mu \mathrm{l}$ of template DNA, and $7.2 \mu \mathrm{l}$ of $\mathrm{H}_{2} \mathrm{O}$. Real-time PCR
(RT-PCR) was performed using a QuantStudio five Real-Time PCR System with the program of $95^{\circ} \mathrm{C}$ for $5 \mathrm{~min}$, and 40 cycles of $95^{\circ} \mathrm{C}$ for $15 \mathrm{~s}$, and $60^{\circ} \mathrm{C}$ for $1 \mathrm{~min}$. A standard curve was derived from serially diluted reference DNA with good linearity $\left(R^{2}>0.98\right)$. Triplicates were applied for each sample, and three 
TABLE 2 | LTL in stroke patients.

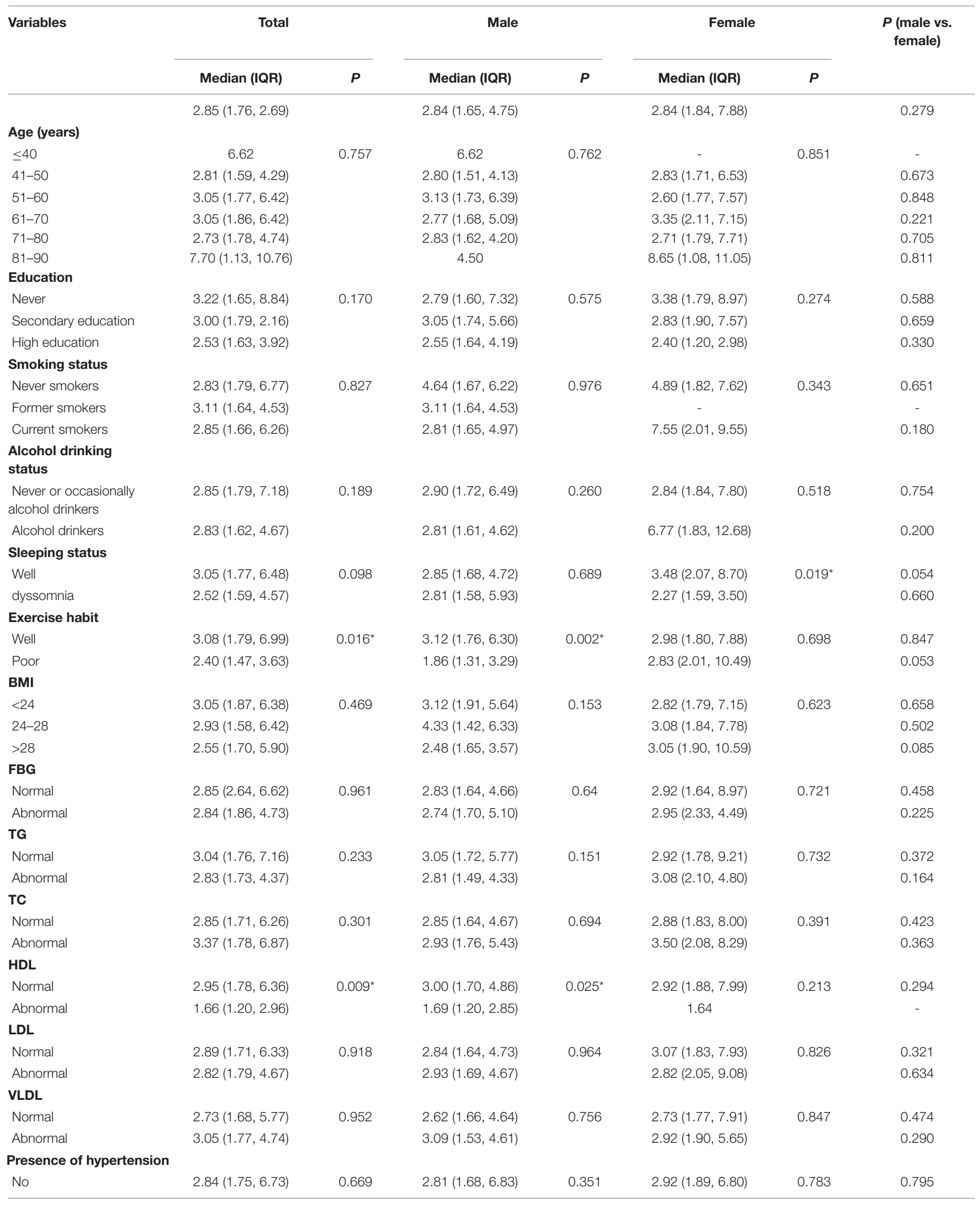


TABLE 2 | (Continued)

\begin{tabular}{|c|c|c|c|c|c|c|c|}
\hline \multirow[t]{2}{*}{ Variables } & \multicolumn{2}{|l|}{ Total } & \multicolumn{2}{|l|}{ Male } & \multicolumn{2}{|l|}{ Female } & \multirow{2}{*}{$\begin{array}{c}P \text { (male vs. } \\
\text { female) }\end{array}$} \\
\hline & Median (IQR) & $P$ & Median (IQR) & $P$ & Median (IQR) & $P$ & \\
\hline \multicolumn{8}{|c|}{ Presence of coronary heart disease } \\
\hline Yes & $2.85(1.74,5.49)$ & & $2.85(1.62,4.54)$ & & $2.84(1.79,8.01)$ & & 0.196 \\
\hline No & $2.82(1.68,5.54)$ & 0.141 & $2.81(1.65,5.54)$ & 0.862 & $2.82(1.79,5.65)$ & 0.051 & 0.775 \\
\hline Yes & $3.72(1.96,8.02)$ & & $3.54(1.80,4.22)$ & & $7.80(2.07,11.76)$ & & 0.072 \\
\hline \multicolumn{8}{|c|}{ Presence of diabetes } \\
\hline No & $3.12(1.72,7.16)$ & 0.140 & $3.05(170,5.37)$ & 0.152 & $3.38(1.77,9.05)$ & 0.424 & 0.299 \\
\hline Yes & $2.81(1.77,3.57)$ & & $2.72(1.43,3.89)$ & & $2.82(2.12,3.53)$ & & 0.348 \\
\hline
\end{tabular}

TABLE 3 | Simple linear regression analysis for LTL.

\begin{tabular}{|c|c|c|c|c|c|c|}
\hline \multirow[t]{3}{*}{ Variable } & \multicolumn{6}{|c|}{ Simple linear regression analysis } \\
\hline & \multicolumn{2}{|c|}{ All $(n=312)$} & \multicolumn{2}{|c|}{ Male $(n=208)$} & \multicolumn{2}{|c|}{ Female $(n=104)$} \\
\hline & $\beta(95 \% \mathrm{Cl})$ & $P$ & $\beta(95 \% \mathrm{Cl})$ & $P$ & $\beta(95 \% \mathrm{Cl})$ & $P$ \\
\hline Age & $0.02(-0.02,0.06)$ & 0.413 & $-0.01(-0.05,0.046)$ & 0.847 & $0.04(-0.04,0.12)$ & 0.304 \\
\hline Education & $-0.36(-0.73,0.02)$ & 0.065 & $-0.28(-0.76,0.19)$ & 0.242 & $-0.32(-1.15,0.51)$ & 0.447 \\
\hline Smoking status & $-0.53(-1.42,0.36)$ & 0.241 & $-0.45(-1.64,0.74)$ & 0.457 & $1.46(-2.20,5.11)$ & 0.431 \\
\hline Alcohol drinking & $-0.48(-1.40 .0 .45)$ & 0.310 & $-0.39(-1.48,0.69)$ & 0.470 & $2.32(-1.34,5.97)$ & 0.211 \\
\hline Dyssomnia & $-0.59(-1.73,0.55)$ & 0.310 & $0.09(-1.42,1.59)$ & 0.910 & $-1.75(-3.60,0.11)$ & 0.065 \\
\hline Lack of exercise & $-1.02(-2.21,1.16)$ & 0.090 & $-1.96(-3.29,-0.63)$ & $0.004^{\star}$ & $1.16(-1.20,3.52)$ & 0.331 \\
\hline $\mathrm{BMI}$ & $0.01(-0.11 .0 .13)$ & 0.880 & $-0.09(-2.25,0.07)$ & 0.260 & $0.15(-0.06,0.35)$ & 0.155 \\
\hline FBG & $-0.16(-0.34,0.02)$ & 0.089 & $-0.13(-0.35,0.08)$ & 0.223 & $0.25(-0.58,0.09)$ & 0.147 \\
\hline TG & $-0.47(0.86,-0.08)$ & 0.018 & $-0.46(-0.88,-0.04)$ & $0.032^{\star}$ & $-0.63(-1.51,0.25)$ & 0.159 \\
\hline HDL & $1.37(-0.38,3.12)$ & 0.126 & $1.41(-0.68,3.49)$ & 0.185 & $0,93(-2.34,4.21)$ & 0.573 \\
\hline LDL & $-0.50(-1.11,0.10)$ & 0.103 & $-0.74(-1.43,0.05)$ & 0.036 & $-0.04(-1.24,1.16)$ & 0.947 \\
\hline VLDL & $-0.09(-0.81,0.64)$ & 0.819 & $-0.24(-1.03,0.55)$ & 0.550 & $0.17(-1.60,1.94)$ & 0.850 \\
\hline Hypertension & $-0.21(-1.11,0.68)$ & 0.640 & $-0.89(-1.92,0.15)$ & 0.093 & $0.94(-0.80,2.68)$ & 0.287 \\
\hline Diabetes & $-1.28(-2.34,-0.23)$ & $0.018^{\star}$ & $-0.85(-2.17,0.47)$ & 0.204 & $-2.19(-4.01,-0.38)$ & $0.019^{\star}$ \\
\hline
\end{tabular}

FBG, fasting blood glucose; TG, triglyceride; TC, total cholesterol; HDL, high-density lipoprotein; LDL, low-density lipoprotein; VLDL, very low-density lipoprotein. Corrected $P$-value was 0.004 by Bonferroni correction. ${ }^{*} P<0.05$.

or more repetitions were performed for each sample. The assay was repeated when the $\mathrm{SD}$ was greater than 0.5 among triplicates.

\section{Statistical Analysis}

Gender-based subgroup analysis was performed. The Kolmogorov-Smirnov goodness-of-fit test was performed to test the normality of continuous data. Continuous data were presented as mean and SD when data were distributed normally, while median and interquartile range (IQR) were presented when data were not distributed normally. Categorical data were presented as frequency. The student's $t$-test was used for the comparison of normally distributed continuous variables. Mann-Whitney $U$ test or Kruskal-Wallis test was used to compare not normally distributed continuous variables. The chisquare test was used for the comparison of frequencies. Fisher's exact test was used when more than $20 \%$ of the cells in any cross-tabulation had an expected count less than or equal to 5. Pearson product-moment correlation analysis was conducted to determine the correlations between LTL and FBG, TG, TC, HDL, or VLDL. Simple linear regression was conducted to determine the single explanatory variable. Multiple linear regression was performed to determine the independent variable. The selection of covariates for male and female patients was based on the following criteria: LTL-related variables reported in previous studies and collected in this study were selected by gender; the frequency of exposure was more than $10 \%$ after correlation analysis and univariate analysis by gender. The frequencies of smoking and alcohol consumption were less than $10 \%$ in female patients. Thus, these two covariates were not included in female patients. Predictors for all stroke patients (Model 1) were 
TABLE 4 | Multiple linear regression analysis for LTL.

\begin{tabular}{|c|c|c|c|c|c|c|}
\hline \multirow[t]{3}{*}{ Model } & \multirow[t]{3}{*}{ Variable } & \multicolumn{5}{|c|}{ Multivariable linear regression analysis } \\
\hline & & All $(n=312)$ & Male $(n=208)$ & Female $(n=104)$ & & \\
\hline & & $\beta$ or $\beta(95 \% \mathrm{Cl})$ & $\beta$ or $\beta(95 \% \mathrm{Cl})$ & $\beta$ or $\beta(95 \% \mathrm{Cl})$ & $P$ & $f^{2}$ \\
\hline 1 & TG & $-0.69(-1.26,-0.11)$ & & & $0.019^{\star}$ & 0.086 \\
\hline \multirow[t]{2}{*}{2} & Lack of exercise & & $-1.80(-3.12,-0.49)$ & & $0.025^{\star}$ & 0.129 \\
\hline & LDL & & $-3.22(-6.05,-0.390)$ & & $0.026^{\star}$ & \\
\hline \multirow[t]{2}{*}{3} & Sleeping status & & & $-2.00(-3.96,-0.07)$ & $0.045^{\star}$ & 0.176 \\
\hline & Diabetes & & & $-2.13(-4.10,-0.27)$ & $0.035^{\star}$ & \\
\hline
\end{tabular}

$\beta$, regression coefficient; $\mathrm{Cl}$, confidence interval; $f^{2}$, Cohen's effect size.

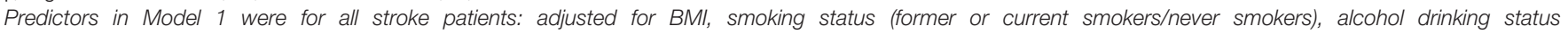

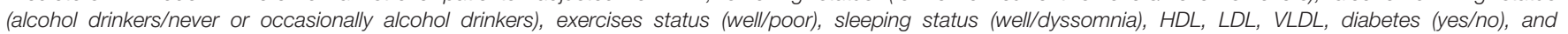
hypertension (yes/no).

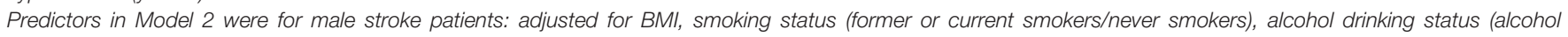
drinkers/never or occasionally alcohol drinkers), sleeping status (well/dyssomnia), TG, HDL, VLDL, diabetes (yes/no), and hypertension (yes/no).

Predictors in Model 3 were for female stroke patients: adjusted for BMI, exercises status (well/poor), TG, HDL, LDL, and hypertension (yes/no).

${ }^{\star} P<0.05$.

adjusted for BMI, smoking status (current smoker or former smoker/non-smoker), alcohol drinking status (current alcohol drinker/non-alcohol drinkers or occasionally alcohol drinker), exercise status (well/poor), dyssomnia (yes/no), HDL, LDL, VLDL, diabetes (yes/no), and hypertension (yes/no). Predictors for male stroke patients (Model 2) were adjusted for BMI, smoking status (current smoker or former smoker/non-smoker), alcohol drinking status (current alcohol drinker/non-alcohol drinkers or occasionally alcohol drinker), dyssomnia (yes/no), TG, HDL, VLDL, diabetes (yes/no), and hypertension (yes/no). Predictors for female stroke patients (Model 3) were adjusted for BMI, exercise status (well/poor), TG, HDL, LDL, and hypertension (yes/no). $P<0.05$ was considered statistically significant. Bonferroni correction was applied for correlation analysis and simple linear regression analysis and corrected $P$-values were presented. Cohen's effect size $f^{2}$ was calculated for the statistical power of multiple linear regression. IBM SPSS Statistics was used for data analysis.

\section{RESULTS}

\section{Characteristics of Participants}

A total of 312 stroke patients were included in the study. Multiple risk factors of stroke were included based on previous studies. The age of patients ranges from 28 to 90 years with an average age of $62.02 \pm 10.70$ years. The mean age of female patients was higher than that of male patients $(p<0.05), 65.52 \pm 10.61$ years vs. $60.27 \pm 10.33$ years (Table 1). Patients aged 50 to 60 years old were the major portion in males accounting for $32.69 \%$. However, patients aged 60 to 70 years old were the major portion in females accounting for $36.54 \%$. Significant differences in education levels, smoking history, drinking status, sleep status, exercise status, hypertension, and diabetes were detected in both male and female groups $(p<0.05)$ (Table 1). Compared with female patients, male patients had more unhealthy drinking and smoking habits, but higher education level and sleep quality. The prevalence of hypertension and diabetes was lower in male patients (Table 1). It indicated that gender difference of multiple factors does exist in stroke patients.

\section{Differences of Leukocyte Telomere Length Based on the Variables}

Leukocyte telomere length was significantly shorter in patients lacking exercise and in patients with low HDL $(P<0.05)$, separately (Table 2). No significant difference was detected in other aspects. The gender-based subgroup analysis was further conducted. LTL was significantly shorter in male patients lacking exercise and with low HDL $(P<0.05)$, separately (Table 2$)$, while LTL was significantly shorter in female patients with dyssomnia $(P<0.05)$. It indicated that LTL may differently associate with risk factors in male and female patients.

Considering medications may contribute to the difference of LTL, the difference of LTL based on medications was analyzed when a medication is taken by more than 10 patients (Zhang et al., 2020; Huang et al., 2021). The medication taken by the patients was summarized in Supplementary Table 1. No difference of LTL was detected based on four medications: calcium channel blocker, biguanides, aspirin, and clopidogrel (Supplementary Table 2). It indicated that medication may have no effect on LTL.

\section{Associations Between Leukocyte Telomere Length and Multiple Risk Factors}

To examine associations between risk factors and LTL, Pearson product-moment correlation analysis and simple linear regression were conducted. The levels of FBG $(r=-0.115$, $P<0.05)$ and TG $(r=-0.134, P<0.05)$ were negatively correlated with LTL in all patients. In male patients, the levels of TG $(r=-0.151, P<0.05)$ and LDL $(r=-0.148, P<0.05)$ were negatively correlated with LTL, separately (Supplementary Table 3). By simple linear regression analysis, LTL was inversely associated with the levels of TG $[\beta(95 \% \mathrm{CI})=-0.47(0.86,-0.08)$, 
$P<0.05]$ and diabetes $[\beta(95 \% \mathrm{CI})=-1.28(-2.34,-0.23)$, $P<0.05]$ in stroke patients. Importantly, LTL was inversely associated with lack of exercise $[\beta(95 \% \mathrm{CI})=-1.96(-3.29$, $-0.63), P<0.05]$ and abnormal TG levels $[\beta(95 \% \mathrm{CI})=-0.46$ $(-0.88,-0.04), P<0.05]$ in male patient, while short LTL was associated with diabetes $[\beta(95 \% \mathrm{CI})=-2.19(-4.01,-0.38)$, $P<0.05$ ] in female patients (Table 3 ). The regression coefficients were not significant when a Bonferroni correction was applied (corrected $P<0.004$ ).

To further identify the independent variables among the risk factors, multiple linear regression analysis was performed. After adjusting confounders, TG remained negatively associated with LTL in all patients $[\beta(95 \% \mathrm{CI})=-0.69(-1.26,-0.11), P=0.019]$ (Table 4). Lack of exercise $[\beta(95 \% \mathrm{CI})=-1.80(-3.12,-0.49)$, $P=0.025]$ and TG levels $[\beta(95 \% \mathrm{CI})=-3.22(-6.05,-0.390)$, $P=0.026]$ were negatively associated with LTL in male patients, while dyssomnia $[\beta(95 \% \mathrm{CI})=-2.00(-3.96,-0.07), P=0.045]$ and diabetes $[\beta(95 \% \mathrm{CI})=-2.13(-4.10,-0.27), P=0.035]$ were negatively associated with LTL in female patients (Table 4). Cohen's effect size $f^{2}$ was reported for statistical power (Table 4).

\section{DISCUSSION}

Leukocyte telomere length is short in patients with stroke. Consistently, cardiovascular risk factors, also correlated with stroke, were associated with short LTL in a case-control study (Alzain et al., 2017). However, the heterogeneity of LTL is high in stroke patients. To minimize the age effect on the heterogeneity, we analyzed LTL in 73 healthy controls and 57 stroke patients within a narrow age range, 50-60 years. No significant difference was detected between healthy controls and patients. Then, the gender effect was investigated. Although the number of female patients is less, the variance of LTL in the female patients is very mild within 50-60 years (Lapham et al., 2015). Different trends were observed in male and female patients. LTL of controls was almost equal to that of male patients, while LTL tended to be short in female patients compared with controls (Supplementary Figure 1). It suggests that gender-specific effects on LTL may exist in stroke patients. Moreover, the importance of gender difference in the disease population has been investigated in a couple of studies (Sullivan et al., 2019). However, no study was performed on stroke patients. Thus, the gender difference of LTL should be investigated in patients with stroke.

In this study, significant differences in demographic characteristics, lifestyles, VLDL levels, and disease history were detected between male and female patients. From a lifestyle aspect, LTL was associated with exercise status in male patients and sleep quality in female patients. However, LTL was not associated with demographic characteristics, disease history, and medications in stroke patients. Importantly, lack of exercise and TG levels were negatively associated with LTL in male patients but not in female patients, while dyssomnia and diabetes were negatively associated with LTL in female patients but not in male patients by multiple regression analysis. Our finding clearly showed that gender difference does exist in associations between risk factors and LTL.
Triglyceride, an independent risk factor, was negatively associated with LTL in male patients but not in female patients. It was reported that high TG is associated with obesity and is a target for obesity treatment (Chen and Farese, 2000). Recent evidence suggests that excess adipose tissue plays a key role in inducing a chronic and systemic inflammatory state contributing to TL shortening in obese individuals (Welendorf et al., 2019). However, no studies have reported gender differences in obesity-associated TL shortening. In this study, we showed that LTL tended to be shorter in male patients than that in female patients when BMI > 28 although it was not significant. Moreover, weight loss has been shown to promote the improvement of chronic inflammation and oxidative stress of adipose tissue and alleviate telomere attrition. It suggests that obesity might be more harmful to men than to women, which might be associated with high TG levels in men. Lack of exercise was associated with short LTL in male patients but not in female patients. Lack of exercise is complemented by housework in female patients as females usually share more housework than males.

Previous reports suggest that women have at least a $40 \%$ increased risk of insomnia compared with men (Guidozzi, 2015). Gender differences in associations between sleep and LTL have not been investigated in any populations although associations between sleep duration, snoring or circadian rhythm disturbance, and LTL were reported (Barceló et al., 2010; Carroll et al., 2016; Shin et al., 2016; Wynchank et al., 2019). Our study showed that sleep quality was associated with LTL in female patients but not in male patients. Diabetes was independently associated with LTL in female patients but not in male patients. It may be associated with the dysregulation of endocrine and metabolism systems, which plays a more pivotal role in females than in males. Our study suggested that the quality of sleep and the well management of diabetes are more critical for female patients.

We acknowledge a number of limitations in this study. First, due to the nature of the cross-sectional study, no causal effect of risk factors on LTL shortening can be concluded although we provided gender differences in associations between risk factors and LTL. Second, our results may be affected by confounders not included in this study such as socioeconomic status and physical activity status in addition to exercises. Moreover, the sample size is limited, particularly for subgroup analysis. The statistical power ranges from a small effect in the total patient group to medium effects in male and female subgroups based on Cohen's effect size $f^{2}$. Although a number of trends were found in our study, they did not reach significance. In addition, racial difference is not considered as only Han Chinese patients were included in this study. The study needs to be improved by increasing sample size, including more factors associated with LTL, recruiting patients of different races, and conducting cohort studies in the future.

\section{CONCLUSION}

In conclusion, our study showed that LTL is differently associated with risk factors in male and female patients with stroke. It 
indicated that gender difference should be considered when LTL is potentially applied as an index of risk and prognosis for stroke. Our study also provides an insight into that gender differences should be considered when developing intervention strategies for stroke prevention and treatment. However, a study with an increased sample size is still necessary.

\section{DATA AVAILABILITY STATEMENT}

The original contributions presented in the study are included in the article/Supplementary Material, further inquiries can be directed to the corresponding author/s.

\section{ETHICS STATEMENT}

The studies involving human participants were reviewed and approved by Research Ethics Committee of Jining Medical University. The patients/participants provided their written informed consent to participate in this study. Written informed consent was obtained from the individual(s) for the publication of any potentially identifiable images or data included in this article.

\section{REFERENCES}

Alzain, M. A., Asweto, C. O., Zhang, J., Fang, H., Zhao, Z., Guo, X., et al. (2017). Telomere length and accelerated biological aging in the china suboptimal health cohort: a case-control study. OMICS 21, 333-339. doi: 10.1089/omi.2017. 0050

Barceló, A., Piérola, J., López-Escribano, H., De La Peña, M., Soriano, J. B., AlonsoFernández, A., et al. (2010). Telomere shortening in sleep apnea syndrome. Respir. Med. 104, 1225-1229. doi: 10.1016/j.rmed.2010.03.025

Blackburn, E. H., Epel, E. S., and Lin, J. (2015). Human telomere biology: a contributory and interactive factor in aging, disease risks, and protection. Science 350, 1193-1198. doi: 10.1126/science.aab3389

Calado, R. T., and Young, N. S. (2009). Telomere diseases. N. Engl. J. Med. 361, 2353-2365.

Cao, W., Li, X., Zhang, X., Zhang, J., Sun, Q., Xu, X., et al. (2019). No causal effect of telomere length on ischemic stroke and its subtypes: a mendelian randomization study. Cells 8:159. doi: 10.3390/cells8020159

Cao, W., Zheng, D., Zhang, J., Wang, A., Liu, D., Zhang, J., et al. (2020). Association between telomere length in peripheral blood leukocytes and risk of ischemic stroke in a Han Chinese population: a linear and non-linear Mendelian randomization analysis. J. Transl. Med. 18:385. doi: 10.1186/s12967020-02551-1

Carroll, J. E., Esquivel, S., Goldberg, A., Seeman, T. E., Effros, R. B., Dock, J., et al. (2016). Insomnia and telomere length in older adults. Sleep 39, 559-564.

Cawthon, R. M. (2002). Telomere measurement by quantitative PCR. Nucleic Acids Res. 30:e47.

Chen, H. C., and Farese, R. V. Jr. (2000). DGAT and triglyceride synthesis: a new target for obesity treatment? Trends Cardiovasc. Med. 10, 188-192. doi: 10.1016/s1050-1738(00)00066-9

Cheng, Y. Y., Kao, T. W., Chang, Y. W., Wu, C. J., Peng, T. C., Wu, L. W., et al. (2017). Examining the gender difference in the association between metabolic syndrome and the mean leukocyte telomere length. PLoS One 12:e0180687. doi: 10.1371/journal.pone.0180687

Choi, E. S., Chang, Y. K., Lee, D. H., Ko, J. H., Lim, I., Bang, H., et al. (2018). Gender-specific associations between quality of life and leukocyte telomere length. Maturitas 107, 68-70. doi: 10.1016/j.maturitas.2017.10.008

Donnan, G. A., Fisher, M., Macleod, M., and Davis, S. M. (2008). Stroke. Lancet $371,1612-1623$.

\section{AUTHOR CONTRIBUTIONS}

$\mathrm{YH}$ and YW contributed to the study design. YQW and FJ performed the experiments, analyzed the data, and wrote the manuscript. RL, XZ, and LY contributed to the data collection and critical comments. $\mathrm{HZ}, \mathrm{QK}, \mathrm{YH}$, and $\mathrm{YW}$ revised the manuscript. All authors reviewed and approved the manuscript.

\section{FUNDING}

This study was funded by the Natural Science Foundation of Shandong Province of China (Grant ZR2018BC022 to FJ), the National Natural Science Foundation of China (Grant 81771147 and 81971019 to YW), and the NSFC cultivation project of Jining Medical University (Grant JYP2018KJ09 to FJ).

\section{SUPPLEMENTARY MATERIAL}

The Supplementary Material for this article can be found online at: https://www.frontiersin.org/articles/10.3389/fnagi. 2021.719538/full\#supplementary-material

Gao, D., Zhang, R., Ji, G., Li, C., Guo, D., Jin, T., et al. (2018). Relative telomere length and stroke risk in a Chinese Han population. J. Mol. Neurosci. 66, 475-481. doi: 10.1007/s12031-018-1160-9

Gardner, M., Bann, D., Wiley, L., Cooper, R., Hardy, R., Nitsch, D., et al. (2014). Gender and telomere length: systematic review and meta-analysis. Exp. Gerontol. 51, 15-27.

Ghimire, S., Hill, C. V., Sy, F. S., and Rodriguez, R. (2019). Decline in telomere length by age and effect modification by gender, allostatic load and comorbidities in National Health and Nutrition Examination Survey (19992002). PLoS One 14:e0221690. doi: 10.1371/journal.pone.0221690

Guidozzi, F. (2015). Gender differences in sleep in older men and women. Climacteric 18, 715-721.

Huang, J., Peng, X., Dong, K., Tao, J., and Yang, Y. (2021). The association between antidiabetic agents and leukocyte telomere length in the novel classification of type 2 diabetes mellitus. Gerontology 67, 60-68. doi: 10.1159/00051 1362

Jin, X., Pan, B., Dang, X., Wu, H., and Xu, D. (2018). Relationship between short telomere length and stroke: a meta-analysis. Medicine (Baltimore) 97:e12489. doi: 10.1097/MD.0000000000012489

Kappei, D., and Londoño-Vallejo, J. A. (2008). Telomere length inheritance and aging. Mech. Ageing Dev. 129, 17-26.

Lapham, K., Kvale, M. N., Lin, J., Connell, S., Croen, L. A., Dispensa, B. P., et al. (2015). Automated assay of telomere length measurement and informatics for 100,000 subjects in the Genetic Epidemiology Research on Adult Health and Aging (GERA) Cohort. Genetics 200, 1061-1072. doi: 10.1534/genetics.115. 178624

Luo, D., Hou, Q., Yu, J., and Yu, D. (2017). Telomere length associated with the risks of high-risk and ischemic stroke in southern Chinese Han population. Oncotarget 8, 105915-105922. doi: 10.18632/oncotarget.2 2509

Needham, B. L., Diez Roux, A. V., Bird, C. E., Bradley, R., Fitzpatrick, A. L., Jacobs, D. R., et al. (2014). A test of biological and behavioral explanations for gender differences in telomere length: the multi-ethnic study of atherosclerosis. Biodemography Soc. Biol. 60, 156-173. doi: 10.1080/19485565.2014.947471

O’Donnell, M. J., Xavier, D., Liu, L., Zhang, H., Chin, S. L., Rao-Melacini, P., et al. (2010). Risk factors for ischaemic and intracerebral haemorrhagic stroke in 22 countries (the INTERSTROKE study): a case-control study. Lancet 376, 112-123. doi: 10.1016/S0140-6736(10)60834-3 
Shadyab, A. H., Lamonte, M. J., Kooperberg, C., Reiner, A. P., Carty, C. L., Manini, T. M., et al. (2017a). Association of accelerometer-measured physical activity with leukocyte telomere length among older women. J. Gerontol. A Biol. Sci. Med. Sci. 72, 1532-1537. doi: 10.1093/gerona/glx037

Shadyab, A. H., Lamonte, M. J., Kooperberg, C., Reiner, A. P., Carty, C. L., Manini, T. M., et al. (2017b). Leisure-time physical activity and leukocyte telomere length among older women. Exp. Gerontol. 95, 141-147.

Shin, C., Yun, C. H., Yoon, D. W., and Baik, I. (2016). Association between snoring and leukocyte telomere length. Sleep 39, 767-772. doi: 10.5665/sleep. 5624

Sullivan, S., Hammadah, M., Al Mheid, I., Shah, A., Sun, Y. V., Kutner, M., et al. (2019). An investigation of racial/ethnic and sex differences in the association between experiences of everyday discrimination and leukocyte telomere length among patients with coronary artery disease. Psychoneuroendocrinology 106, 122-128. doi: 10.1016/j.psyneuen.2019.03.021

Tian, Y., Wang, S., Jiao, F., Kong, Q., Liu, C., and Wu, Y. (2019). Telomere length: a potential biomarker for the risk and prognosis of stroke. Front. Neurol. 10:624. doi: 10.3389/fneur.2019.00624

Welendorf, C., Nicoletti, C. F., Pinhel, M. A. S., Noronha, N. Y., De Paula, B. M. F., and Nonino, C. B. (2019). Obesity, weight loss, and influence on telomere length: new insights for personalized nutrition. Nutrition 66, 115-121. doi: 10.1016/j.nut.2019.05.002

Wynchank, D., Bijlenga, D., Penninx, B. W., Lamers, F., Beekman, A. T., Kooij, J. J. S., et al. (2019). Delayed sleep-onset and biological age: late sleep-onset is associated with shorter telomere length. Sleep 42:zsz139. doi: 10.1093/sleep/ zsz139

Zhang, S., Li, R., Yang, Y., Chen, Y., Yang, S., Li, J., et al. (2020). Longitudinal association of telomere attrition with the effects of antihypertensive treatment and blood pressure lowering. Aging Dis. 11, 494-508. doi: 10.14336/AD.2019. 0721

Conflict of Interest: The authors declare that the research was conducted in the absence of any commercial or financial relationships that could be construed as a potential conflict of interest.

Publisher's Note: All claims expressed in this article are solely those of the authors and do not necessarily represent those of their affiliated organizations, or those of the publisher, the editors and the reviewers. Any product that may be evaluated in this article, or claim that may be made by its manufacturer, is not guaranteed or endorsed by the publisher.

Copyright (c) 2021 Wang, Jiao, Zheng, Kong, Li, Zhang, Yan, Hao and Wu. This is an open-access article distributed under the terms of the Creative Commons Attribution License (CC BY). The use, distribution or reproduction in other forums is permitted, provided the original author(s) and the copyright owner(s) are credited and that the original publication in this journal is cited, in accordance with accepted academic practice. No use, distribution or reproduction is permitted which does not comply with these terms. 